\title{
Silver-Coated Gold Nanoparticles as Concentrating Probes and Matrices for Surface-Assisted Laser Desorption/Ionization Mass Spectrometric Analysis of Aminoglycosides
}

\author{
Ming-Ta Wang, ${ }^{\text {a Ming-Han Liu, }}{ }^{\text {b C. R. Chris Wang, }}{ }^{\text {b }}$ and \\ Sarah Y. Chang ${ }^{a, c}$ \\ a Department of Chemistry, Tunghai University, Taichung, Taiwan \\ b Department of Chemistry and Biochemistry, National Chung Cheng University, Chia-Yi, Taiwan \\ c Department of Chemistry, National Kaohsiung Normal University, Kaohsiung, Taiwan
}

\begin{abstract}
A novel method for the determination of aminoglycosides by surface-assisted laser desorption/ ionization mass spectrometry (SALDI MS) with the aid of silver-coated gold nanoparticles (Au@AgNPs) has been developed. The Au@AgNPs with surface capped by anionic citrate were used as concentrating probes as well as matrices in SALDI MS. Adsorption of aminoglycosides onto the nanoparticles was mainly through electrostatic attraction. The aminoglycoside-adsorbed nanoparticles were directly characterized by SALDI MS after a simple washing. Using Au@AgNPs to preconcentrate the aminoglycosides from $500 \mu \mathrm{L}$ buffer solution, the limits of detection (LODs) at signal-to-noise ratio of 3 were 3, 25, 15, 30, and 38 $\mathrm{nM}$ for paromomycin, kanamycin A, neomycin, gentamicin, and apramycin, respectively. This method was successfully applied to the determination of aminoglycosides in human plasma samples. The LODs of aminoglycosides in plasma samples were 9, 130, 81, and $180 \mathrm{nM}$ for paromomycin, kanamycin A, neomycin, and gentamicin, respectively. Recoveries of aminoglycosides in plasma samples were about 80\%. (J Am Soc Mass Spectrom 2009, 20, 1925-1932) (O 2009 American Society for Mass Spectrometry
\end{abstract}

$\mathrm{R}$ ecently, surface-assisted laser desorption/ionization mass spectrometry (SALDI MS) has become a popular technique due to the rapid development of nanomaterials. Nanoparticles have been recognized as effective SALDI matrices because of their large surface area-to-volume ratio, ease of sample preparation, flexibility of sample deposition, and no dependence on the irradiation wavelength. Gold and Nile-red adsorbed gold nanoparticles were used as SALDI matrix for the mass spectrometric determination of aminothiols [1], neutral small carbohydrates [2], and adenosine triphosphate [3]. Carbon nanotubes [4], magnetic iron oxide [5], silver [6], gold [7], and $\mathrm{TiO}_{2}$-coated magnetic nanoparticles [8] were also employed for the analysis of peptides and proteins. The mass range could be extended to $24 \mathrm{KDa}$ with the use of nanoparticles as matrices, and the detection limit for peptide is about 20 fmol. In addition, titanium dioxide [9], silver [10], and silicon nanoparticles [11] successfully desorbed and

Address reprint requests to Professor S. Y. Chang, Department of Chemistry, National Kaohsiung Normal University, No. 62, Shenjhong Rd., Yanchao Township, Kaohsiung County 824, Taiwan. E-mail: ychang@nknucc. nknu.edu.tw, and to Professor C. R. Chris Wang, Department of Chemistry and Biochemistry, National Chung Cheng University, Min-Hsiung, Chia-Yi 621, Taiwan. E-mail: checrw@ccu.edu.tw ionized enediol compound, olefin, and small molecules, respectively. The use of nanoparticles as SALDI matrices not only eliminates matrix ion interference but also improves sample homogeneity. Sweet-spot searching is not required, therefore better shot-to-shot and sampleto-sample reproducibility is obtained.

To improve the sensitivity and minimize the interferences of sample matrix, the nanoparticles were also used as concentrating probes to separate and concentrate the analytes from complex biologic samples before SALDI MS analysis. The surface of the nanoparticle was modified for the selective retention of analyte while removing interferences through simple washing. The collected analyte-adsorbed nanoparticles were either analyzed directly by SALDI/MS or mixed with conventional organic matrices, such as sinapinic acid (SA) and $\alpha$-cyano-4-hydroxycinnamic acid (CHCA), for their matrix-assisted laser desorption/ionization (MALDI) MS analysis. Various approaches were employed for selectively binding the target analytes. For examples, $\mathrm{C}_{18}$ functionalized silica [12] and $\mathrm{C}_{8}$-functionalized magnetic nanoparticles [13] utilized hydrophobic interactions to extract peptides from solutions. Gold nanoparticles [14, 15], diamond [16], bare silica [17], and oleate-modified iron oxide nanoparticles [18] were also 
used to selectively concentrate positively charged proteins through electrostatic interaction. In addition, the ZnO-poly (methyl methacrylate) nanobeads concentrated peptides mainly through hydrogen bonding and dipole-dipole interaction [19]. These approaches allow the MS detection of low concentration of peptides and proteins in complex matrix solution.

Aminoglycosides are an important class of antibiotics that are active against both gram-positive and gramnegative bacterial infections, and they have found widespread use in both human and veterinary medicine. However, the use of aminoglycosides may cause sideeffects of ototoxicity and nephrotoxicity [20,21]. Careful monitoring of the aminoglycosides in plasma is routinely used as a guide to dosing, to prevent toxicity and to ensure efficacy. Our goal in this study is to examine whether the Au@AgNPs can be used to capture the aminoglycosides in dilute and complex sample solution. The surfaces of the Au@AgNPs were capped with citrate, which contains anionic carboxylate groups at $\mathrm{pH}>4$. The citrate-capped Au@AgNPs could attract positively charged aminoglycosides through electrostatic attraction. The ability of the Au@AgNPs to act as both concentrating probes and assisting material in SALDI MS was evaluated. The applicability of the method to the determination of aminoglycosides in human plasma was also demonstrated. To our knowledge, this is the first example that demonstrates the use of Au@AgNPs as concentrating probe and matrix for the analysis of aminoglycosides in SALDI MS.

\section{Experimental}

\section{Chemicals}

Kanamycin sulfate, gentamicin sulfate, paromomycin sulfate, apramycin sulfate, neomycin sulfate, potassium carbonate, and gold(III) chloride trihydrate, SA were purchased from Sigma (St. Louis, MO, USA). Hydroquinone and tannic acid powder (extra pure) were obtained from Riedel-de Haën (Seelze, Germany). Silver nitrate was purchased from J and J Materials (Neptune City, NJ, USA). Hydrogen tetrachloroaurate(III) trihydrate was obtained from Acros Organics (Morris Plains, NJ, USA). CHCA and 2,5-dihydroxybenzoic acid (DHB) were obtained from Aldrich (Milwaukee, WI, USA), and trisodium citrate dihydrate was purchased from Showa (Tokyo, Japan). All chemicals were used as received without further purification. All other chemicals were of reagent grade. Water purified with a Barnstead NANOpure system (Dubuque, IA, USA) was used for all solutions.

\section{Synthesis of Silver Nanoparticles}

To prepare negatively charged silver nanoparticles (AgNPs) with a control in size, we decided to take a seed-mediated growth method and to use trisodium citrate as the capping molecules. In the first step, gold nanoparticles (AuNPs) were prepared using the stan- dard citrate reduction method [22]. An aqueous solution $(79 \mathrm{~mL})$ containing $4 \mathrm{~mL}$ of $1 \%$ trisodium citrate dihydrate, $3 \mathrm{~mL}$ of $1 \%$ tannic acid, and $25 \mathrm{mM} \mathrm{K}_{2} \mathrm{CO}_{3}$ (final concentration) was heated to $60^{\circ} \mathrm{C}$, and then hydrogen tetrachloroaurate (III) solution $(1 \%, 1 \mathrm{~mL})$ was added to the solution while stirring. Both solutions were preheated to $60{ }^{\circ} \mathrm{C}$ under vigorous stirring before mixing. The color change of the solution from bright yellow to crimson indicated the formation of monodispersed AuNPs. The AuNPs were believed to be capped with anionic citrate ions, which stabilize particle dispersity. The resulting colloidal gold particles were used as the seeds for the second-step silver growth. We conducted a modified procedure for catalytic growth of silver on gold, reported previously [23, 24]. The size of the final Au@AgNPs could be controlled by adjusting the silver coating conditions, such as time and its precursor concentration. An aliquot of $60 \mu \mathrm{L}$ of $0.1 \mathrm{M}$ $\mathrm{AgNO}_{3}$ aqueous solution was added into $5 \mathrm{~mL}$ colloidal gold solution. The resulting mixture was dropwise added into $110 \mu \mathrm{L} 0.01 \mathrm{M}$ hydroquinone solution under stirring. We adjusted the solution $\mathrm{pH}$ to 11 , to increase the reducing rate by adding $\mathrm{NaOH}$ aqueous solution. The same silver coating procedure was repeated three times to form final Au@AgNPs in desired average diameter as well as in a good size control.

\section{Characterizations of Silver Nanoparticles}

The size and distribution of either AuNPs or Au@AgNPs were characterized through transmission electron microscopy (TEM) measurements using a JOEL-2010 TEM from JOEL (Tokyo, Japan) at an accelerating $200 \mathrm{kV}$. TEM samples were prepared by dipping 200 mesh Formvar coated copper grid into the nanoparticle solutions. The successful coating of silver on colloidal gold was confirmed by the UV/VIS absorption spectroscopy (HP8453; Hewlett-Packard, Palo Alto, CA, USA) with a $0.5 \mathrm{~cm}$ path-length sample cell. The citrate conjugation on the Au@AgNPs surface was confirmed by FT-IR spectroscopy (FT-IR-430, JASCO, Tokyo, Japan).

\section{Sample Preparation and Extraction Procedure}

Aqueous standard solutions containing $1 \mathrm{mM}$ aminoglycosides were prepared in water, and diluted to desired concentration by D. I. water or $10 \mathrm{mM}$ phosphate buffer. The aminoglycoside solutions were stored at $-20^{\circ} \mathrm{C}$. CHCA, SA, and DHB solutions, both fresh prepared in 1:1:0.001 acetonitrile/water/TFA solution, were used as the MALDI matrices for aminoglycosides. MALDI samples were prepared by mixing $1 \mu \mathrm{L}$ of analyte solution and $1 \mu \mathrm{L}$ of matrix solution on the target and letting them dry at room temperature.

The Au@AgNPs solution $(100 \mu \mathrm{L})$ and aminoglycoside solution (500 $\mu \mathrm{L})$ were added to the $1.5 \mathrm{~mL}$ sample vial. The mixture was thoroughly mixed with a shaker for $1 \mathrm{~h}$. After centrifugation at 28,600 $\mathrm{g}$ for $10 \mathrm{~min}$ (MIKRO22R; Hettich Zentrifugen, Tuttlingen, Germany), 
the supernatant was removed. The collected nanoparticles were washed with $100 \mu \mathrm{L}$ of D. I. water to remove impurities. Then the analyte-adsorbed nanoparticles were deposited on a stainless steel target and allowed to dry.

\section{Preparation of Plasma Samples}

Blood sample was centrifuged immediately to obtain the plasma, which was stored $-20{ }^{\circ} \mathrm{C}$ until analysis. An aliquot of $500 \mu \mathrm{L}$ plasma sample was deproteinized by adding 1000 $\mu \mathrm{L}$ of acetonitrile. After centrifugation at $6000 \mathrm{~g}$ for $15 \mathrm{~min}$, $450 \mu \mathrm{L}$ of the supernatant liquid was spiked with $50 \mu \mathrm{L}$ of aminoglycoside solution. Plasma samples of various aminoglycoside contents were similarly prepared by spiking the plasma with the desired amount of aminoglycoside. Blank plasma sample was prepared by spiking $50 \mu \mathrm{L}$ of D. I. water to $450 \mu \mathrm{L}$ of the supernatant liquid. The $\mathrm{pH}$ value of plasma samples was adjusted to 5 by the addition of concentrated $(1 \mathrm{M}) \mathrm{HCl}$. Then the aminoglycosidespiked plasma $(500 \mu \mathrm{L})$ was mixed with Au@AgNPs, following the procedure described above. The recoveries of aminoglycosides in plasma were estimated by spiking $50 \mu \mathrm{L}$ of aminoglycosides standards to $450 \mu \mathrm{L}$ of plasma sample before deproteinization.

\section{SALDI-TOF MS}

Mass spectrometry experiments were performed in the positive-ion mode on a reflectron-type time-of-flight mass spectrometer (Microflex; Bruker Daltonics, Billerica, MA, USA) with a flight length of $1.9 \mathrm{~m}$. The samples were irradiated with a $337 \mathrm{~nm}$ nitrogen laser at $20 \mathrm{~Hz}$. The generated ions were accelerated at voltage of $19 \mathrm{kV}$. To obtain good signal-to-noise ratios, the laser energy was adjusted to slightly higher than the threshold, and each spectrum was acquired by an average of 50 laser pulses.

\section{Results and Discussion}

\section{Characterization of $A g N P S$}

The silver nanoparticles were prepared by the seedmediated growth process. Dispersive AuNPs were chosen to be the seed material, which provided an easier control in the final size and the uniformity of silver particles than that of bare silver nanoparticle (AgNPs) synthesis. The TEM image showed that the average diameter of the prepared AuNP seed is of $9 \pm 1 \mathrm{~nm}$, and the final Au@AgNPs from the seed mediated growth possessed a mean diameter of $39 \pm 5 \mathrm{~nm}$. To routinely characterize the Au@AgNPs, UV-VIS absorption spectroscopy was used to ensure proper silver coating on the AuNP seed. The absorption spectrum of the AuNP seed exhibited a typical surface plasmon resonance (SPR) band located at $523 \mathrm{~nm}$. After the seed mediated growth process, the spectrum showed a distinct absorption spectral band at $410 \mathrm{~nm}$, which was attributed to the characteristic SPR band of bare silver nanoparticles and indicated the successful silver coating on the sur- face of AuNP seeds. The capping citrate and its free form originally in AuNP seed solution are also believed to provide similar stabilization effect on thus prepared Au@AgNPs. Citrate-capped Au@AgNPs may trap positively charged aminoglycosides through ionic interaction. The citrate conjugation on the Au@AgNPs surface was confirmed by FT-IR spectroscopy. The absorption peak at $1634 \mathrm{~cm}^{-1}$ corresponds to the $\mathrm{C}=\mathrm{O}$ absorption band from the carboxylic acid group. To estimate the number of particles per unit volume, we followed the previously reported procedure [25]. The concentration of the Au@AgNPs used in this study was determined to be $1.8 \times 10^{13}$ particles $/ \mathrm{L}$.

\section{$A u @ A g N P s$ as Assisted Matrices in SALDI/MS}

The aminoglycosides contain two or more aminosugars linked by glycosidic bonds to an aminocyclitol component. To test the ability of using Au@AgNPs as matrices, we conducted SALDI/MS using paromomycin as a representative analyte. Figure 1 a shows the mass spectra of paromomycin with ion signals at $m / z 638.78$ and 654.77, which correspond to $[\mathrm{M}+\mathrm{Na}]^{+}$and $[\mathrm{M}+\mathrm{K}]^{+}$, respectively. The alkali ion adducts were commonly observed when using nanomaterials as SALDI matrices. Although some background ions were contributed by $[\mathrm{Ag}]^{+},[2 \mathrm{Ag}]^{+}$, and $[3 \mathrm{Ag}]^{+}$, the results indicated that Au@AgNPs as SALDI matrices could be applied to the detection of aminoglycosides. To minimize the formation of the potassium adduct ion of paromomycin, which had a detrimental effect on the sensitivity, we dissolved paromomycin in phosphate buffer $(10 \mathrm{mM}$, $\mathrm{pH} 5)$. Figure $1 \mathrm{~b}$ indicates that paromomycin produced $[\mathrm{M}+\mathrm{Na}]^{+}$as the major ions in the mass spectrum. As a result of fewer adduct ions, the average intensity of the $[\mathrm{M}+\mathrm{Na}]^{+}$was 2.8 times greater than that in D. I. water over seven sample spots. For comparison, the mass spectra for paromomycin using DHB as MALDI matrix is shown in Figure 1c. Paromomycin produced $[\mathrm{M}+\mathrm{H}]^{+}$as the major ions in the mass spectrum; a signal was also present for paromomycin cationized by sodium. The signal intensities of $[\mathrm{M}+\mathrm{H}]^{+}$when using DHB were lower than the signal intensities of $[\mathrm{M}+$ $\mathrm{Na}^{+}$obtained using Au@AgNPs as matrices (Figure 1b). Other commonly used MALDI matrices, such as CHCA and SA, were also utilized for paromomycin, but no signal was obtained.

The sample preparation is also an important step for the separation and enrichment of trace amounts of analytes from complex biologic samples before SALDI/MS analysis. The citrate-capped Au@AgNPs possess negative charges on their surfaces, thus they have the ability to bind positively charged species in aqueous solutions through electrostatic attraction. The amount of Au@AgNPs has great effect on the sensitivity of the method. Various amounts $(50-500 \mu \mathrm{L})$ of Au@AgNPs were mixed with $500 \mu \mathrm{L}$ of $1 \mu \mathrm{M}$ paromomycin solution, and the mixtures were diluted to a total volume of $1 \mathrm{~mL}$. The optimum amount of 


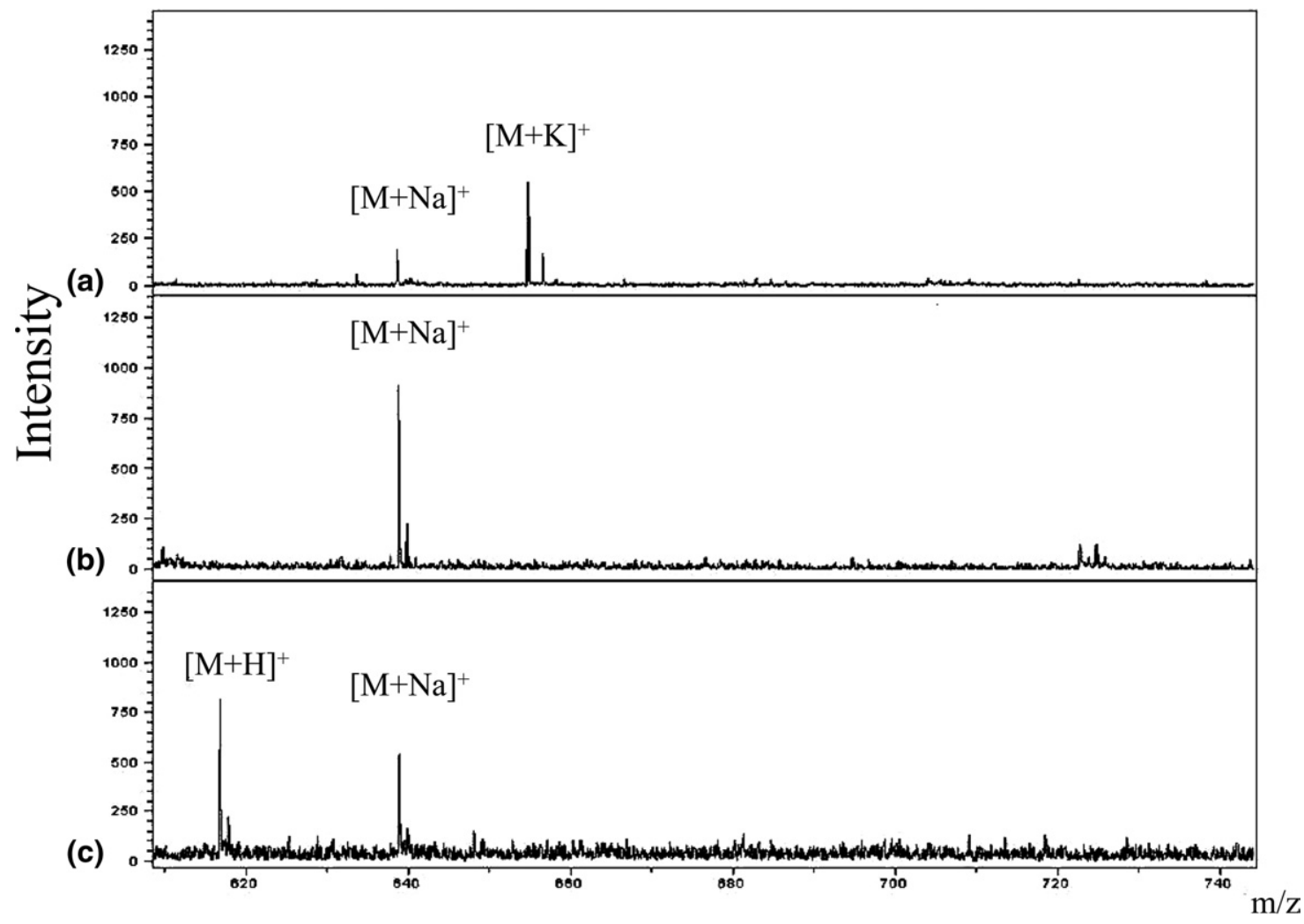

Figure 1. Mass spectra of paromomycin with matrices of (a) Au@AgNPs, (b) Au@AgNPs, (c) DHB, respectively. The sample solutions were prepared in D. I. water (a) and (c), and in $10 \mathrm{mM}$ phosphate buffer, pH 5 (b). The peaks at $m / z 616.82,638.78$, and 654.77 are assigned to the $[\mathrm{M}+\mathrm{H}]^{+},[\mathrm{M}+\mathrm{Na}]^{+}$, and $[\mathrm{M}+\mathrm{K}]^{+}$ions. The concentration of paromomycin was $10 \mu \mathrm{M}$. A total of 50 pulsed laser shots were applied under a laser set at $30 \mu \mathrm{J} /$ pulse.

the Au@AgNPs was $100 \mu \mathrm{L}$. At lower amount of Au@AgNPs, low background and analyte ion intensities were generated because insufficient energy was absorbed and the ionization efficiency was low. In addition, the capture capability was low at lower amount of Au@AgNPs. Basically the capture capability is high at higher amount of Au@AgNPs; however, the number of adsorbed molecules in each Au@AgNPs is lower if analyte is limited. As a result, poor sensitivity was usually obtained at higher amount of Au@AgNPs. Recent work demonstrated that gold NPs could be used as SALDI matrix when used at ratio of $10^{4}-10^{7}$ analyte molecules to $1 \mathrm{NP}[1,7]$. In our experiment, $100 \mu \mathrm{L}$ of the Au@AgNPs solution was estimated to contain $1.8 \times$ $10^{9}$ particles. The ratio of analyte to Au@AgNPs was calculated to be $1.7 \times 10^{5}$. Compared with conventional MALDI matrix, the ratio of analyte to Au@AgNPs is much higher. This result suggests that Au@AgNPs have the capacity to ionize more than one analyte per laser pulse, or they have the potential to regenerate to a matrix-"active" state between each laser pulse.

\section{Optimization of Extraction Efficiency}

To optimize the extraction efficiency of Au@AgNPs, five major aminoglycosides were chosen as the model analytes, including kanamycin A, paromomycin, neo- mycin, gentamicin, and apramycin. The extraction efficiencies of aminoglycosides were monitored based on the absolute ion intensities of sodium adduct ions in three samples. The effect of the $\mathrm{pH}$ of solution on the analyte ion signals is illustrated in Figure 2. The citric acid dissociates into carboxylate, and the surface of Au@AgNPs carries negative charges at $\mathrm{pH}>4$. By increasing the $\mathrm{pH}$ value from 4 to 5 , the signal intensities of the five aminoglycosides increase because of the increases of negative charges on the Au@AgNPs surface (pKa values of the citric acid are 3.2, 4.4, and 5.7 [1]. The maximum adsorption all occurred at $\mathrm{pH} 5$ for the five aminoglycosides. The $\mathrm{pKa}$ values of the five aminoglycosides are 5.5-9.5 [26-28]; therefore the aminoglycosides carried 4-6 positive charges at $\mathrm{pH} 5$. With increasing the $\mathrm{pH}$ of solution from 5 to 10 , the amount of analyte adsorbed on the Au@AgNPs and the analyte ion signals decreased. This is due to the decrease of positive charges on the aminoglycosides. At $\mathrm{pH} 10$, most of the five aminoglycosides were neutral molecules and were not extracted efficiently by the negatively charged Au@AgNPs. These observations suggest that electrostatic attraction is the main binding interaction between aminoglycosides and $\mathrm{Au} @ \mathrm{AgNPs}$. In addition, the hydrophobic interaction and hydrogen bonding may also be involved in the adsorption process. Figure 3 shows the mass spectrum obtained using 


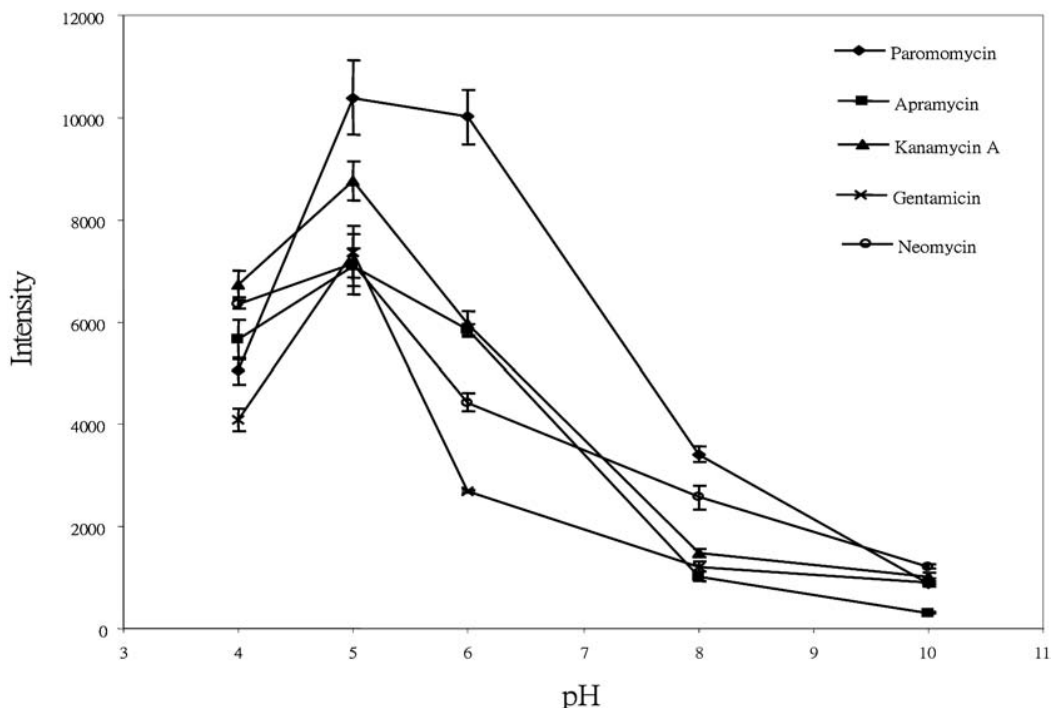

Figure 2. Ion intensities of aminoglycosides after extraction by the Au@AgNPs at different pH values. The concentrations of aminoglycosides were $1 \mu \mathrm{M}$. Au@AgNPs was used as the SALDI matrix. A total of 50 pulsed laser shots were applied under a laser set at $30 \mu \mathrm{J} / \mathrm{pulse}$.

Au@AgNPs to extract the five aminoglycosides from 10 $\mathrm{mM}$ phosphate buffer ( $\mathrm{pH} 5)$. The signals were observed at $m / z$ 500.68, 507.61, 562.72, 637.78, and 638.78, which correspond to the sodium adduct ions of gentamicin, kanamycin A, apramycin, neomycin, and paromomycin, respectively.

Using Au@AgNPs as selective and concentrating probes, we expected that the sensitivities of the aminoglycosides could be enhanced in the SALDI/MS analysis. Figure 4a shows the mass spectrum of paromomycin $(5 \mu \mathrm{M})$ without the preconcentration process. The signal/noise $(\mathrm{S} / \mathrm{N})$ ratio was determined to be 3 . No signals were obtained when the paromomycin concentration was lower than $5 \mu \mathrm{M}$.
With the use of Au@AgNPs to preconcentrate the paromomycin from $500 \mu \mathrm{L}$ buffer solution (10 mM phosphate, $\mathrm{pH} 5)$, the signal was greatly enhanced as shown in Figure $4 \mathrm{~b}$. Figure $4 \mathrm{c}$ shows the mass spectrum of $10 \mathrm{nM}$ paromomycin with preconcentration of paromomycin by Au@AgNPs. With the preconcentration strategy, the sensitivity of paromomycin was increased by more than two orders of magnitude.

\section{Quantitative Analysis of Aminoglycosides}

The homogeneity achieved using nanoparticles for SALDI/MS is higher than obtained by the formation

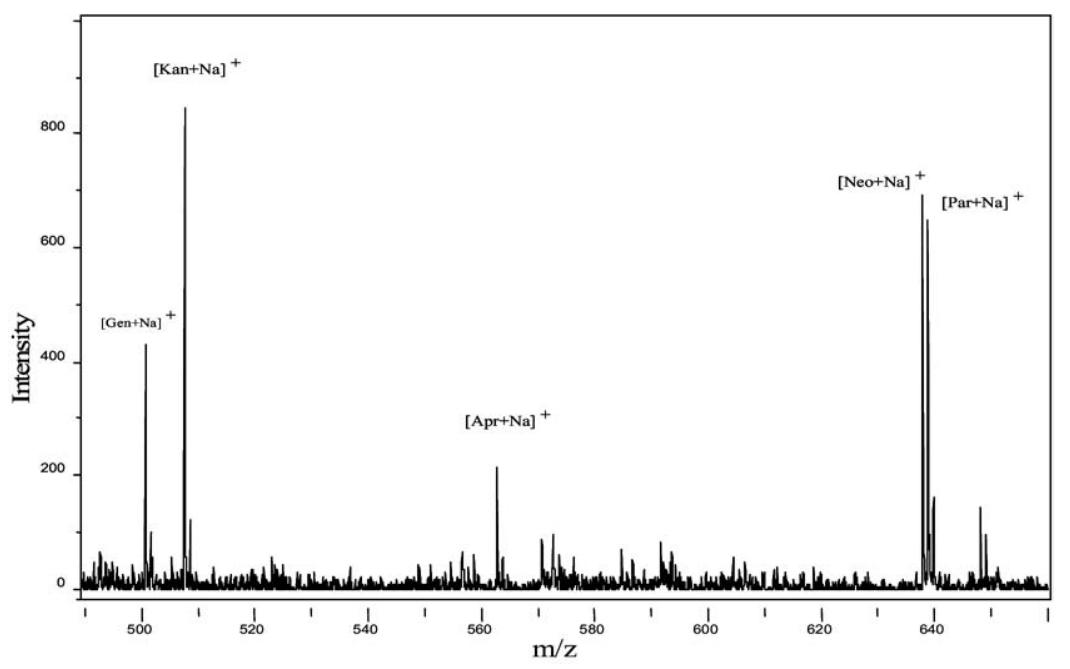

Figure 3. Mass spectra was obtained from $500 \mu \mathrm{L}$ mixture solution (10 mM phosphate buffer, $\mathrm{pH}$ 5) of gentamicin, kanamycin A, apramycin, neomycin, and paromomycin. The peaks at $\mathrm{m} / \mathrm{z} 500.68$, $507.61,562.72,637.78$, and 638.78 are assigned to the sodium adduct ions of gentamicin, kanamycin A, apramycin, neomycin and paromomycin, respectively. The concentrations of gentamicin, kanamycin $\mathrm{A}$, apramycin, and neomycin were $0.4 \mu \mathrm{M}$, and the concentration of paromomycin was $0.2 \mu \mathrm{M}$. Other conditions as in Figure 2. 


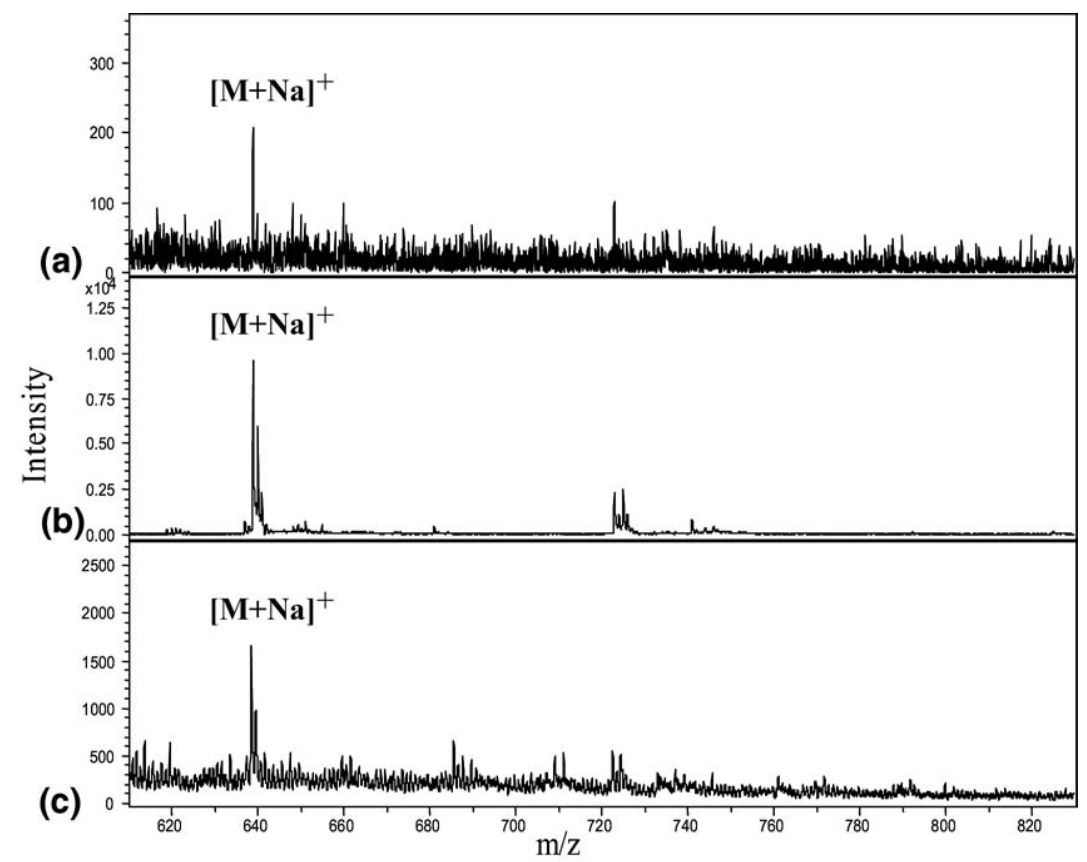

Figure 4. Mass spectra of paromomycin obtained (a) without any pretreatment $(5 \mu \mathrm{M})$, and (b) with preconcentration of $5 \mu \mathrm{M}$, and (c) $10 \mathrm{nM}$ paromomycin solution by Au@AgNPs. Other conditions as in Figure 2.

of crystals of the analyte with the conventional organic matrix. We also examined the reproducibility of the sample preparation. The intensities of sodium adduct ions of aminoglycosides varied less than $8 \%$ over 21 sample spots (three samples). Calibration curves for kanamycin A, neomycin, gentamicin, and apramycin in aqueous solution were constructed over the concentration range 0.1 to $10 \mu \mathrm{M}$. For paromomycin, the concentration range of calibration curve was $10 \mathrm{nM}$ to $10 \mu \mathrm{M}$. Within the concentration range studied, a good linear correlation $(\gamma>0.99)$ between signal intensities and concentrations was obtained for each analyte. The linear range covered two orders of magnitude of concentration. The concentration LODs were calculated based on an $\mathrm{S} / \mathrm{N}$ ratio of 3 , which are $3,25,15,30$, and $38 \mathrm{nM}$ for paromomycin, kanamycin $\mathrm{A}$, neomycin, gentamicin, and apramycin, respectively. The LODs of the aminoglycosides obtained in the present study are comparable to those obtained by the LC/MS/MS coupled with solidphase extraction [29].

\section{Mass Analysis of Aminoglycosides in Human Plasma}

To evaluate the applicability of the method for biologic and clinical analysis, human plasma spiked with paromomycin, kanamycin A, neomycin, and gentamicin was used as the test sample. Analysis of aminoglycosides in plasma is often problematic. Since AgNPs can attract proteins and peptides as well, the extraction efficiency may be affected by the endogenous plasma components. To minimize protein interferences in the preconcentration process and suppression of aminoglycosides ionization, the plasma samples were deproteinized by the addition of acetonitrile, followed by centrifugation. Typical mass spectra obtained from blank and spiked plasma samples are shown in Figure 5. The ions at $m / z 500.68,507.61,637.78$, and 638.78 corresponded to the sodium adduct ions of gentamicin, kanamycin A, neomycin, and paromomycin, respectively. Although the mass spectrum of blank plasma sample contains some extraneous peaks, probably from the endogenous plasma components, they do not interfere with the signals of aminoglycosides.

The calibration curves showed good linearity over the concentration range of $0.3-30 \mu \mathrm{M}$ for kanamycin $\mathrm{A}$, neomycin, gentamicin, and apramycin, and $30 \mathrm{nM}-30$ $\mu \mathrm{M}$ for paromomycin. This range sufficiently covers the therapeutic range of the four aminoglycosides in plasma $(2-10 \mu \mathrm{g} / \mathrm{mL})$ [29]. At $\mathrm{S} / \mathrm{N}$ ratio of 3, the LODs for paromomycin, kanamycin A, neomycin, and gentamicin in plasma were calculated to be $9,130,81$, and 180 $\mathrm{nM}$, respectively. Due to matrix effect, the LODs were about 3 to 6 times higher than that in standard solution. Recoveries of aminoglycosides from plasma were determined by spiking $450 \mu \mathrm{L}$ of plasma with $50 \mu \mathrm{L}$ of aminoglycoside standards before deproteinization, followed by extraction by Au@AgNPs and SALDI/MS analysis. Based on triplicate measurements, the mean recoveries of paromomycin, kanamycin $\mathrm{A}$, neomycin, and gentamicin were found to be $81 \%, 78 \%, 82 \%$, and $77 \%$, respectively. 


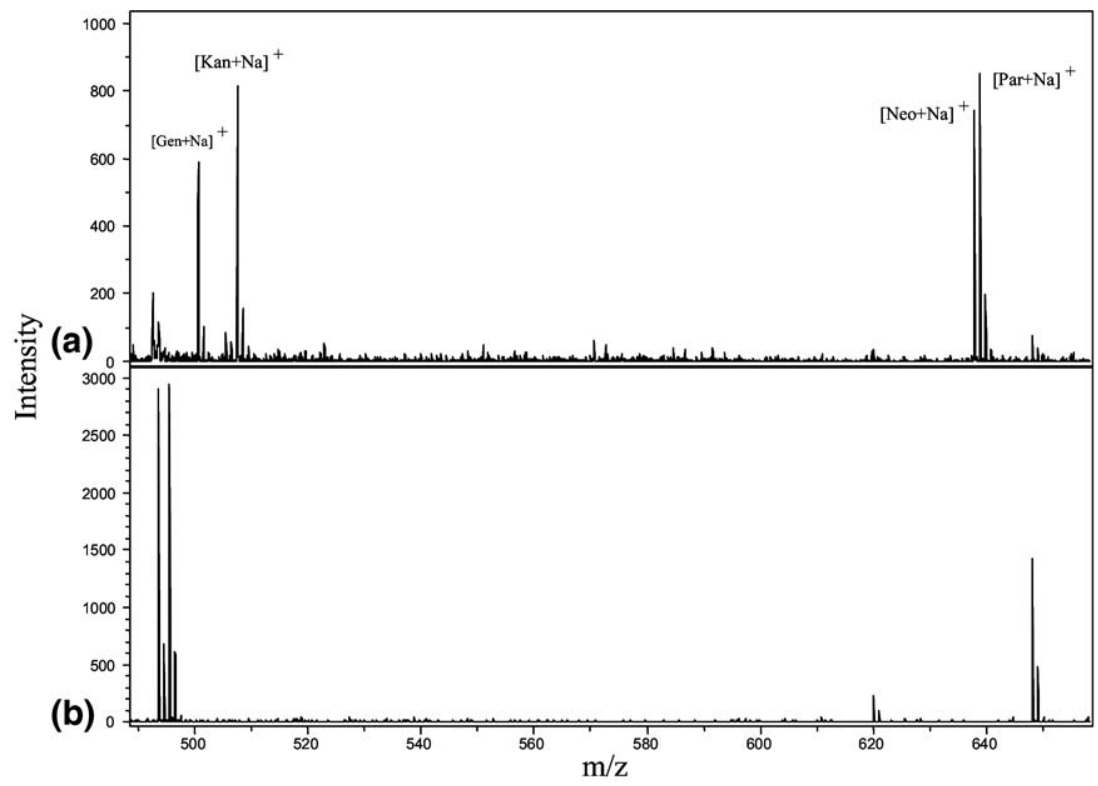

Figure 5. Mass spectra of human plasma samples. (a) Plasma spiked with $0.5 \mu \mathrm{M}$ gentamicin, kanamycin A, neomycin, and paromomycin; (b) blank. The ions at $\mathrm{m} / \mathrm{z} 500.68,507.61,637.78$, and 638.78 are assigned to the sodium adduct ions of gentamicin, kanamycin $\mathrm{A}$, neomycin, and paromomycin, respectively. Other conditions as in Figure 2.

\section{Conclusions}

The citrate-capped Au@AgNPs were used as concentrating probes and matrices for the determination of aminoglycosides through SALDI/MS. This SALDI/MS method, with its speed and ease of operation, is suitable for high-throughput screening of aminoglycosides in human plasma samples.

\section{Acknowledgments}

The authors acknowledge financial support for this work by the National Science Council (NSC 97-2113-M-017-002-MY2) of Taiwan.

\section{References}

1. Huang, Y.-F.; Chang, H.-T. Nile Red-Adsorbed Gold Nanoparticle Matrixes for Determining Aminothiols through Surface-Assisted Laser Desorption/Ionization Mass Spectrometry. Anal. Chem. 2006, 78, 14851493.

2. Su, C.-L.; Tseng, W.-L. Gold Nanoparticles as Assisted Matrix for Determining Neutral Small Carbohydrates through Laser Desorption/ Ionization Time-of-Flight Mass Spectrometry. Anal. Chem. 2007, 79, 1626-1633.

3. Huang, Y.-F.; Chang, H.-T. Analysis of Adenosine Triphosphate and Glutathione through Gold Nanoparticles Assisted Laser Desorption/ Ionization Mass Spectrometry. Anal. Chem. 2007, 79, 4852-4859.

4. Chen, W.-Y.; Wang, L.-S.; Chiu, H.-T.; Chen, Y.-C.; Lee, C.-Y. Carbon Nanotubes as Affinity Probes for Peptides and Proteins in MALDI MS Analysis. J. Am. Soc. Mass Spectrom. 2004, 15, 1629-1635.

5. Chen, W.-Y.; Chen, Y.-C. Affinity-Based Mass Spectrometry Using Magnetic Iron Oxide Particles as the Matrix and Concentrating Probes for SALDI MS Analysis of Peptides and Proteins. Anal. Bioanal. Chem. 2006, 386, 699-704.

6. Hua, L.; Chen, J.; Ge, L.; Tan, S. N. Silver Nanoparticles as Matrix for Laser Desorption/Ionization Mass Spectrometry of Peptides. J. Nanopart. Res. 2007, 9, 1133-1138.

7. McLean, J. A.; Stumpo, K. A.; Russel, D. H. Size-Selected (2-10 nm) Gold Nanoparticles for Matrix Assisted Laser Desorption Ionization of Peptides. J. Am. Chem. Soc. 2005, 127, 5304-5305.

8. Chen, C.-T.; Chen, Y.-C. $\mathrm{Fe}_{3} \mathrm{O}_{4} / \mathrm{TiO}_{2}$ Core/Shell Nanoparticles as Affinity Probes for the Analysis of Phosphopeptides Using $\mathrm{TiO}_{2}$
Surface-Assisted Laser Desorption/Ionization Mass Spectrometry. Anal. Chem. 2005, 77, 5912-5919.

9. Lee, K.-H.; Chiang, C.-K.; Lin, Z.-H.; Chang, H.-T. Determining Enediol Compounds in Tea Using Surface-Assisted Laser Desorption/Ionization Mass Spectrometry with Titanium Dioxide Nanoparticle Matrices. Rapid Commun. Mass Spectrom. 2007, 21, 2023-2030.

10. Sherrod, S. D.; Diaz, A. J.; Russell, W. K.; Cremer, P. S.; Russell, D. H. Silver Nanoparticles as Selective Ionization Probes for Analysis of Olefins by Mass Spectrometry. Anal. Chem. 2008, 80, 6796-6799.

11. Wen, X.; Dagan, S.; Wysocki, V. H. Small-Molecule Analysis with Silicon-Nanoparticle-Assisted Laser Desorption/Ionization Mass Spectrometry. Anal. Chem. 2007, 79, 434-444.

12. Turney, K.; Drake, T. J.; Smith, J. E.; Tan, W.; Harrison, W. W. Functionalized Nanoparticles for Liquid Atmospheric Pressure MatrixAssisted Laser Desorption/Ionization Peptide Analysis. Rapid Commun. Mass Spectrom. 2004, 18, 2367-2374.

13. Yao, N.; Chen, H.; Lin, H.; Deng, C.; Zhang, X. Enrichment of Peptides in Serum by C8-Funtionalized Magnetic Nanoparticles for Direct Matrix-Assisted Laser Desorption/Ionization Time-of-Flight Mass Spectrometry Analysis. J. Chromatogr. A 2008, 1185, 93-101.

14. Tseng, C.-H.; Ho, K.-C.; Lin, Y.-S.; Chen, Y.-C. Gold Nanoparticles as Selective and Concentrating Probes for Samples in MALDI MS Analysis. Anal. Chem. 2004, 76, 4337-4342.

15. Vanderpuile, B. N. Y.; Han, G.; Rotello, V. M.; Vachet, R. W. Mixed Monolayer-Protected Gold Nanoparticles as Selective Peptide Extraction Agents for MALDI-MS Analysis. Anal. Chem. 2006, 78, 5491-5496.

16. Kong, X. L.; Huang, L. C. L.; Hsu, C.-M.; Chen, W.-H.; Han, C.-C.; Chang, H.-C. High-Affinity Capture of Proteins by Diamond Nanoparticles for Mass Spectrometric Analysis. Anal. Chem. 2005, 77, 259-265.

17. Agrawal, K.; Wu, H.-F. Bare Silica Nanoparticles as Concentrating and Affinity Probes for Rapid Analysis of Aminothiols, Lysozyme and Peptide Mixtures Using Atmospheric-Pressure Matrix-Assisted Laser Desorption/ Ionization Ion Trap and Matrix-Assisted Laser Desorption/Ionization Time-of-Flight Mass Spectrometry. Rapid Commun. Mass Spectrom. 2008, 22, 283-290.

18. Chang, S. Y.; Zheng, N.-Y.; Chen, C.-S.; Chen, C.-D.; Chen, Y.-Y.; Wang, C. R. C. Analysis of Peptides and Proteins Affinity-Bound to Iron Oxide Nanoparticles by MALDI MS. J. Am. Soc. Mass Spectrom. 2007, 18, 910-918.

19. Shen, W.; Xiong, H.; Xu, Y.; Cai, S.; Lu, H.; Yang, P. ZnO-Poly(methyl methacrylate) Nanobeads for Enriching and Desalting Low-Abundant Proteins Followed by Directly MALDI-TOF MS Analysis. Anal. Chem. 2008, 80, 6758-6763.

20. Vandamme, E. J. Biotechnology of Industrial Antibiotics, Marcel Dekker, Inc.: New York and Basel, 1984; p. 329.

21. Stead, D. A. Current Methodologies for the Analysis of Aminoglycosides. J. Chromatogr. B 2000, 747, 69-93.

22. Slot, J. W.; Geuze, H. J. A New Method of Preparing Gold Probes for Multiple-labeling Cytochemistry. Eur. J. Cell. Biol. 1985, 38, 87-93. 
23. Danscher, G. A Revised Version of the Sulphide Silver Method Suitable for both Light and Electron Microscopy. Histochemistry 1981, $71,1-16$.

24. Scopsi, L.; Larsson, L.-I. Effect of Double Application of Antibodies and of Silver Intensification on Immunogold and Peroxidase-Antiperoxidase Staining Techniques. Histochemistry 1985, 82, 321-329.

25. Chou, C.-H.; Chen, C.-D.; Wang, C. R. C. Highly Efficient, WavelengthTunable, Gold Nanoparticle Based Optothermal Nanoconvertors. J. Phys. Chem. B 2005, 109, 11135-11138.

26. Baudoux, P.; Bles, N.; Lemaire, S.; Mingeot-Leclercq, M.; Tulkens, P. M.; Van Bambeke, F. Combined Effect of $\mathrm{pH}$ and Concentration on the
Activities of Gentamicin and Oxacillin Against Staphylococcus aureus in Pharmacodynamic Models of Extracellular and Intracellular Infections. J. Antimicrob. Chemother. 2007, 59, 246-253.

27. Serrano, J. M.; Silva, M. Trace Analysis of Aminoglycoside Antibiotics in Bovine Milk by MEKC with LIF detection. Electrophoresis 2006, 27, 4703-4710.

28. Walter, F.; Vicens, Q.; Westhof, E. Aminoglycoside-RNA Interactions. Curr. Opin. Chem. Biol. 1999, 3, 694-704.

29. Oertel, R.; Neumeister, V.; Kirch, W. Hydrophilic Interaction Chromatography Combined with Tandem-mass Spectrometry to Determine Six Aminoglycosides in Serum. J. Chromatogr. A 2004, 1058, 197-201. 